\title{
Etude De Surcreusement Des Pistes Rurales Ou Forestières, Sur Le Plateau Des Cataractes: Cas Des Routes Koubola - Kibossi Et Koubola - Bissinza (République Du Congo)
}

\author{
Dr. Léonard Sitou, Maitre-assistant \\ Idriss M'bouka Milandou, Doctorant
}

Laboratoire de géographie physique,

Faculté des Lettres, des Arts et des Sciences Humaines (FLASH)

Université Marien Ngouabi, Brazzaville, République du Congo

doi: 10.19044/esj.2017.v13n21p116 URL:http://dx.doi.org/10.19044/esj.2017.v13n21p116

\begin{abstract}
This article investigates the track overdeepening, a rare type of degradation that turns roads into trenches. He carried on two rural tracks south of Brazzaville, in the Pool Department, in the Republic of Congo, quantifying the phenomenon by the cubing method; describes the processes of formation and evolution through in situ observations; Highlights the vulnerability of the environment through the quantification of some variables representing its physical characteristics and, with the ArcGis 10.1 software, maps the phenomenon for a geo-referenced location of the main forms. Estimated at 13,724 tons of land loss on the first axis and 14,424 tons on the second road, the overcrowding is one of the forms of dramatic erosion of agricultural or forest tracks in the study area. Triggered by the bad treatment of the ruts, the processes of installation and evolution of this type of gully are favored by the sensitivity of the tracks themselves marked by apparent densities which vary between 1.7 and $1.8 \mathrm{~g} /$ centimeter cubic, with an average porosity (about 30\%) and that of the geological formations in the clayey-clay ensemble with a high clay content (over 30\%) which favors the process of overdeepening marked by the verticality or almost of the boundary walls. The mean of the erosivity index $\mathrm{R}$ is estimated at 7756,55 MJ.mm/ha.h.an, with an average annual height of $1400 \mathrm{~mm}$ characterizing the climatic aggressiveness of the zone and the slopes which reach and exceed the $25 \%$, Are all aggravating factors. The prediction of this phenomenon requires a change in the method of treatment of the ruts and the stabilization of the forms already constituted and active, by that of the rolling surface and especially the walls by bio-mechanical methods.
\end{abstract}


Keywords: Republic of Congo, Pool, tracK erosion, rut, overcrowding

\section{Résumé}

Cet article étudie le surcreusement de piste, un type de dégradation rare qui transforme les routes en tranchées. Portant sur deux pistes rurales, au sud de Brazzaville, dans le département du Pool, en République du Congo, il quantifie le phénomène par la méthode de cubage; décrit les processus de formation et d'évolution grâce à des observations in situ; met en évidence la vulnérabilité du milieu à travers la quantification de quelques variables représentant ses caractéristiques physiques et, avec le logiciel ArcGis 10.1, cartographie ledit phénomène pour une localisation géo référencée des principales formes. Estimés à 13724 tonnes de pertes en terre sur le premier axe, et à 14424 tonnes sur la deuxième route, le surcreusement constitue une des formes d'érosion spectaculaire des pistes agricoles qui limite la circulation des hommes et des biens, impactant ainsi négativement le développement socioéconomique des populations de la zone d'étude. Déclenché par le mauvais traitement des ornières, les processus d'évolution de cette érosion sont favorisés par la sensibilité des pistes elles-mêmes marquées par des densités apparentes qui varient entre 1,7 et $1,8 \mathrm{~g} / \mathrm{cm}^{3}$ avec une porosité moyenne (environ 30\%) et celle des formations géologiques dans l'ensemble sablo-argileuses avec un taux d'argile qui dépasse les $33 \%$. La moyenne de l'indice d'érosivité $\mathrm{R}$ estimée à 7 756,55 MJ.mm/ha.h.an, avec une hauteur moyenne annuelle de $1400 \mathrm{~mm}$ de pluies, caractérisant l'agressivité climatique de la zone et les pentes qui atteignent et dépassent les $25 \%$, sont autant de facteurs aggravant. La prévision de ce phénomène passerait par un changement de méthode de traitement des ornières. La stabilisation des formes déjà constituées et actives nécessiterait un traitement de la surface de roulement et surtout des parois par des méthodes biomécaniques.

Mots clés: République du Congo, Pool, érosion de pistes, surcreusement, ornières

\section{Introduction}

Au Congo, la rapidité avec laquelle se dégradent les pistes agricoles surtout non bitumées constitue aujourd'hui une grande préoccupation, aussi bien pour les décideurs politiques et administratifs que pour les populations qui sont les principaux utilisateurs et les scientifiques qui cherchent à comprendre le phénomène. Ceci est autant évident que ces axes de communications sont vitaux pour l'économie en général et pour la survie des communautés rurales en particulier. Animée par l'érosion hydrique, cette 
dégradation se manifeste généralement par des rigoles, des ravines générées par les ornières et se transforment en ravins. Certains se forment sur les côtés et remontent par érosion régressive en direction de la route qu'ils coupent plus ou moins perpendiculairement. Aujourd'hui, se développe un type particulier de dégradation qui affecte les pistes en terre dans le département du Pool en général et sur le plateau des Cataractes en particulier : c'est le surcreusement de piste. Celui-ci se manifeste par un approfondissement de lapiste qui se transforme en une tranchée sur des dizaines, voire des centaines de mètres de long avec une profondeur dépassant parfois $5 \mathrm{~m}$. Une fois formée, la tranchée devient un long couloir qui laisse très peu de marge de manœuvre aux automobiles en cas d'enlisement ou d'embourbement à l'intérieur. Cette situation rend les sections de pistes affectées impraticables et paralyse la circulation.

C'est pourquoi un surcreusement entraîne généralement la déviation de piste sur plusieurs kilomètres, provoquant parfois l'enclavement de villages entiers. Par ailleurs, de ces surcreusements sortent souvent d'importants volumes d'eau de ruissellement qui se déversent sur les versants et contribuent aux ravinements sus évoqués. C'est pourquoi en aval de ces formes, les pistes sont généralement plus dégradées. A ce propos, Makanzu Imwangana et al. (2014) pensent que dans la plupart des cas, les ravins observés le long des pistes agricoles ont une relation directe avec lesdites pistes. De ce fait, les surcreusements non seulement compliquent les déplacements de la population, mais sont un facteur aggravant de l'érosion dans la zone d'étude. Bien que répandu dans le département, ce phénomène n'a fait l'objet jusqu'ici, d'aucune recherche scientifique dans cette région. Cette étude analyse les facteurs hydrogéologiques qui sont à l'origine des surcreusements des pistes des villages de Koubola - Bissinza et KoubolaKibossi, dans la sous-Préfecture de Goma tsé-tsé au sud de Brazzaville. Elle dimensionne ces surcreusements et quantifie les pertes de terre sur ces deux pistes. Mais l'étude présente d'abord le cadre général sur lequel elle s'inscrit.

\section{Cadre de l'étude}

Les pistes Koubola-Kibossi et Koubola-Bissinza s'étendent entre $4^{\circ} 10^{\prime} 0^{\prime \prime}$ et $4^{\circ} 30^{\prime} 0^{\prime \prime}$ de latitude sud et entre $14^{\circ} 50^{\prime} 0^{\prime \prime} 15^{\circ} 20^{\prime} 0^{\prime}$ 'de longitude est, à environ $20 \mathrm{~km}$ au sud-ouest de Brazzaville, dans la sous-Préfecture de Goma tsé-tsé, dans le département du Pool (Figure 1). La première, longue d'environ $15 \mathrm{~km}$, commence sur la route nationale $\mathrm{n}^{\circ} 1(\mathrm{RN} 1)$ à l'entrée du village Koubola, avant le nouveau poste de péage et se termine à la gare de Kibossi, sur le Chemin de Fer Congo Océan (CFCO). Elle relie donc deux axes de communications majeures de ce département : la RN1 et le CFCO. La deuxième piste est, quant à elle, longue de près de $11 \mathrm{~km}$. Elle prolonge la première piste en direction du sud- est, traverse deux grands villages, Mayala 
et Moumbouanissa, et aussi Bissinza (fin du secteur de l'étude du tronçon) où elle décrit une boucle en direction de Nganga Loungolo au nord, sur un autre axe routier (Figure 1). C'est une route de raccourcis, qui permet aux agriculteurs, aux exploitants des forêts et aux fabricants de charbon de bois, de joindre Brazzaville en moins de temps, pour la vente de leurs produits. Ces deux pistes d'une importance socio-économique dans la région, traversent un milieu géomorphologique stable mais sensible en raison de ses caractéristiques physiques. En effet, aménagées en plein cœur du plateau des Cataractes, ces pistes traversent un relief très tourmenté fait de collines hautes et convexes atteignant en moyenne $450 \mathrm{~m}$ de hauteur entourées de vallées drainées (M'bouka Milandou, 2009). Ce plateau qui s'étend jusqu'en RDC est taillé dans deux assises géologiques qui se superposent : à la base, la série de l'Inkisi, constituée de grès durs d'âge précambrien et, au sommet, la série des plateaux Batékés formée de sables ocre jaunes d'âge tertiaire (Sautter, 1969). Les grès de l'Inkisi affleurent au fond de certaines vallées où l'enfoncement de certains cours d'eau a permis de les atteindre. Les sols formés à partir de ces roches sont ferralitiques fortement désaturés, avec des taux de matière organique oscillant autour de 2 à $3 \%$ (Denis, 1974). Ils sont, avec les formations superficielles issues de la déségrégation des grès de l'Inkisi, très meubles et sensibles à l'érosion pluviale par l'effet splash et au ravinement par les ruissellements (M'bouka Milandou, 2009).

Le climat est de type bas-congolais (Samba Kimbata, 1978) caractérisé par une alternance d'une saison sèche de 4 mois (juin-septembre) et d'une saison de pluie de 8 mois (octobre à mai). Le réseau hydrographique est dense; il est constitué du fleuve Djoué et de ses affluents qui sont des petites rivières et ruisseaux dont le fonctionnement est tributaire du climat local. Ce réseau hydrographique, bien drainé au cours de la période pluvieuse, charrient de grandes quantités de débris détritiques arrachés en amont par l'érosion; mais en l'absence de pluie et pendant la saison sèche, le volume des eaux diminue et la traversée à gué de certains de ces cours d'eau redevient facile. Quant à la végétation, elle est une mosaïque de forêts ombrophiles occupant surtout les fonds de vallées et de savanes tantôt hautes tantôt basses mais en général arbustive avec Anonna arenaria, Bridelia ferruginéa et l'Hymenocardia-acida comme principales strates ligneuses (Descoings, 1975). 


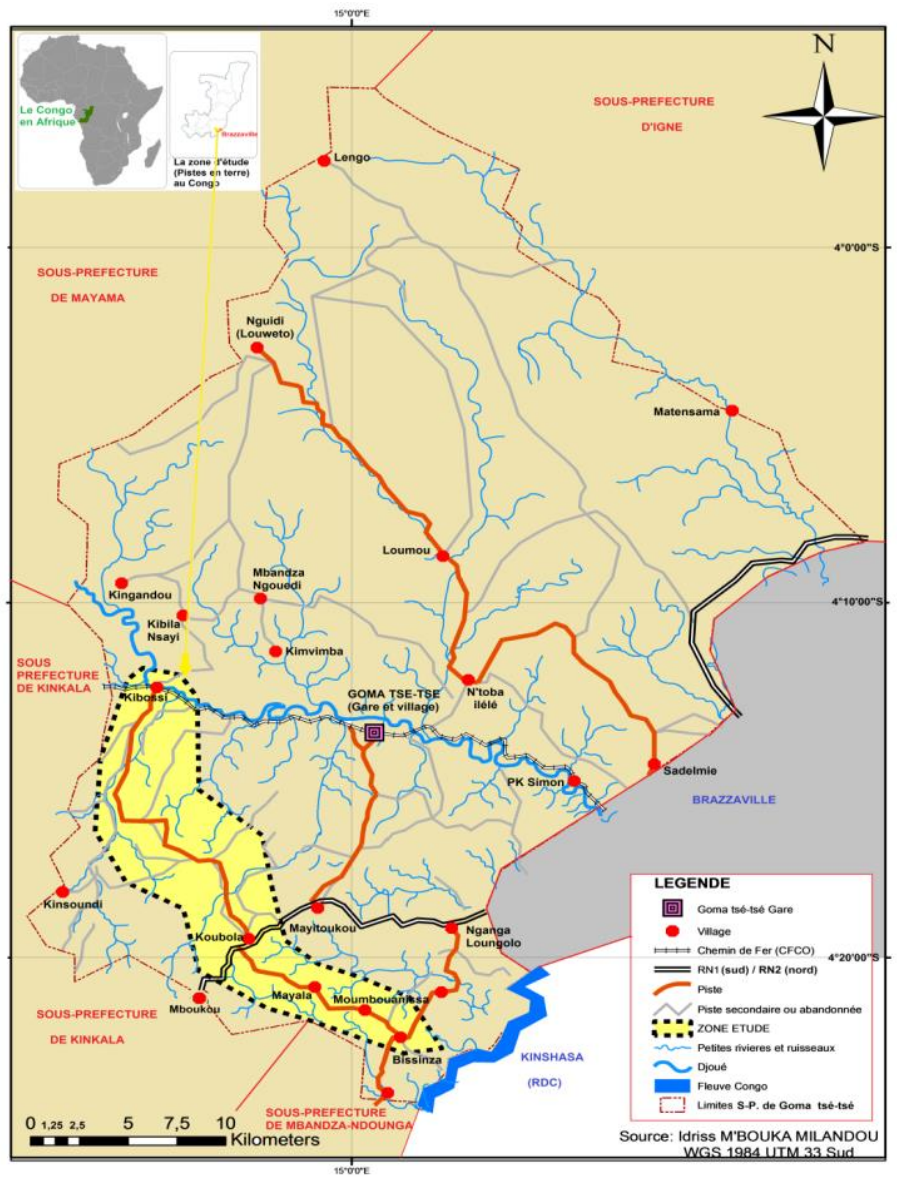

Figure 1 : Localisation des pistes Koubola-Kibossi et Koubola-Bissinza

Ainsi, le cadre physique présente une certaine sensibilité qui a été vérifiée par un certain nombre de méthodes.

\section{Méthodes et données utilisées}

\section{Méthodes qualitatives et quantitatives utilisées}

Des observations de terrain et des investigations documentaires ont été effectuées. Aussi, 14 surcreusements ont-ils été identifiés et quantifiés, soit 8 sur l'axe Koubola-Kibossi et 6 sur la piste Koubola-Bissinza. Un rouleau de corde en nylon, graduée en mètre et un double décamètre ont été utilisés pour le dimensionnement des surcreusements. Ainsi, la largeur, la base, la profondeur et la longueur des excavations ont été mesurées pour obtenir les volumes des formes. A partir de ces derniers, les pertes en terre ont été évaluées. Les mesures ont été effectuées par section, en tenant compte des formes géométriques que prennent les surcreusements. Deux formes 
principales ont été étudiées : les prismes trapézoïdaux et rectangulaires. Les formules suivantes ont été utilisées :

Volume d'un prisme trapézoïdale $: l_{\text {moy. } 1} \neq l_{\text {moy. } 1}$

$V=\left(\frac{l_{\text {moy. } 1}+l_{\text {moy.1 }}^{\prime}}{2} x P_{\text {moy. }}\right) x L_{t}$

$V=$ volume en $\mathrm{m}^{3} ; l_{\text {moy. } 1}=$ largeur moyenne du toit (ouverture) en $\mathrm{m}$;

$l_{\text {moy.l }}^{\prime}=$ largeur moyenne de la base rétrécie en $m ; P_{\text {moy. }}=$ profondeur moyenne en $m ; L_{t}=$ Longueur totale en $m$

Volume d'un prisme rectangulaire : $l_{\text {moy. } 1}=l_{\text {moy. } 1}=l_{\text {moy. }}$.

$V=\left(l_{\text {moy. }} x P_{\text {moy. }}\right) x L_{t .}$ (2)

$V=$ volume en $\mathrm{m}^{3} ; l_{\text {moy. }}=$ largeur moyenne de l'ouverture et de la base en $m$;

$L_{\text {moy. }}=$ Longueur moyenne totale en $m ; P_{\text {moy. }}=$ profondeur moyenne en $\mathrm{m}$; $L_{t .}=$ Longueur totale en $m$

Profondeur moyenne des formes:

$\boldsymbol{P}_{\text {moy. }}=\frac{p_{1}+p_{1}^{\prime}}{2}$

$P_{\text {moy. }}=$ profondeur moyenne en $m$;

$P_{1}=$ profondeur de la paroi $x$ (talus $X$ ) en $m ; P^{\prime}{ }_{1=}$ profondeur de la paroi $y$ (talus opposé) en $m$

\section{Pertes en terre}

$\mathbf{P}=\int \mathbf{x} \mathbf{V}$ (4)

$\int$ comme densité apparente du materiel rémanié $(\mathrm{MR})=$ densité apparente (da) en $\mathrm{g} / \mathrm{cm}^{3}$ et $\mathrm{V}$ : volume totale de chaque forme en $\mathrm{m}^{3}$

\section{Evaluation de la sensibilité du milieu}

La sensibilité du milieu vis-à-vis de l'érosion a été appréciée d'une part, par les caractéristiques de la surface de roulement, notamment la densité apparente du matériel remanié (MR) constituant la surface de roulement (SR); la largeur de la piste et, d'autre part, par la composition texturale des sols autour des pistes et l'agressivité du climat. Les pistes en étude sont taillées directement sur la roche en place. La couche de roulement est constituée du MR par les passages répétés des usagers de la route. La détermination de la densité apparente $(d a)$ de MR a permis d'apprécier la compaction et la porosité de la SR.

A cet effet, 6 échantillons du MR ont été prélevés sur les pistes, à différentes sections, par la méthode de cylindre de $100 \mathrm{~cm}^{3}$ de volume. Ces échantillons, séchés pendant 24 heures à l'étuve à $105^{\circ} \mathrm{C}$, ont été ensuite 
pesés pour obtenir la masse sèche. Connaissant le volume du MR qui est celui du cylindre et sa masse sèche, la densité apparente $(d a)$ du MR a été déterminée avec la formule :

$d a=\frac{P}{V}(\mathbf{5})$

où $\mathrm{P}$ est le poids sec de l'échantillon $(\mathrm{g})$ et $\mathrm{V}$ le volume de l'échantillon prélevé et séché $\left(\mathrm{cm}^{3}\right)$

La largeur des pistes a été mesurée par section, avec un double décamètre. Sur la piste Koubola-Kibossi, sur $15 \mathrm{~km}$ environ de piste, $7 \mathrm{Km}$ ont fait l'objet de mesures sur des sections de $1 \mathrm{~km}$. Sur l'axe KoubolaBissinza, $5 \mathrm{Km}$ ont été mesurés. Le but est d'évaluer la largeur moyenne des pistes, qui influe sur la concentration des ruissellements. Il importe de noter que plus la route est étroite, plus les ruissellements sont concentrés et le risque d'érosion élevé.

La mesure granulométrique apermis de caractériser la texture des sols autour des pistes; elle a été réalisée avec la méthode de tamisage au laboratoire de la société des BTP Andrade Gutierrez. Elle a porté sur 18 échantillons prélevés directement sur les parois des surcreusements à $15 \mathrm{~cm}$, $50 \mathrm{~cm}$ et $100 \mathrm{~cm}$ de profondeur. Trois surcreusements de chaque piste ont fait l'objet d'échantillonnage, soit 3 échantillons par surcreusement à raison d'un échantillon par niveau de profondeur. La mesure granulométrique a permis d'obtenir les taux de sables, de limon et d'argile contenus dans ces surcreusements. Egalement, la teneur en matière organique (MO) contenue dans ces échantillons a été évaluée avec la méthode de perte au feu (PAF) ou d'incinération. Les moyennes des 18 échantillons ont été utilisées à cet effet. Les données pédologiques collectées, traitées et analysées ont été ensuite cartographiées par ArcGis 10.1.

l'Aviation Civile (ANAC). La formule appliquée est la méthode simplifiée de Renard et Freimund (1994) cités par Douay et Lardieg (2010), qui intègre uniquement la hauteur des pluies annuelles moyennes (P). Avec le P> 850 mm à Maya-Maya, cette formule simplifiée s'écrit :

$\mathbf{R}=\mathbf{5 8 7 , 8}-\mathbf{1 , 2 1 9} \mathbf{P}+\mathbf{0 , 0 0 4 1 0 5} \mathrm{P}^{2}(6)$
$\mathrm{R}$ : indice d'érosivité ; P : précipitation annuelle $(\mathrm{mm})$.

Pour analyser les résultats obtenus après évaluation de l'indice R, nous avons utilisé la classification établie par Douay et Lardieg (2010) (Tableau 1) 
Tableau 1 : classification de l'indice d'érosivité (R) par rapport à la vulnérabilité des sols

\begin{tabular}{|c|c|c|c|}
\hline Classe & \multicolumn{2}{|c|}{ Erosivité $(\mathrm{R})$} & Vulnérabilité des sols \\
\hline 4 & $>5000$ & Forte érosivité & Très élevée \\
\hline 3 & 4000 à 5000 & & Elevée \\
\hline 2 & 3000 à 4000 & & Modérée \\
\hline 1 & 2000 à 3000 & & Faible \\
\hline 0 & 0 à 2000 & Faible érosivité & Très faible \\
\hline
\end{tabular}

Source : Douay et Lardieg, 2010

\section{La cartographie du surcreusement}

Les coordonnées géographiques ( $\mathrm{X}$ et $\mathrm{Y}$ ) relevées sur chaque forme lors des mesures de terrain, ont été insérées dans ArcGis 10.1 sur un fond de carte de la zone d'étude au $1: 250000^{\mathrm{e}}$ fournie par le Centre de Recherches Géographiques et Cartographiques (CERGEC) en 2014, scannée et convertie en format Grid. Le géoréférencement de la carte sur ArcCatalog a été suivi de sa digitalisation sur ArcMap, afin de créer les couches des 2 pistes, des villages et de l'hydrographie. Une couche de localisation des surcreusements sur les pistes a été conçue en transférant les coordonnées géographiques de chaque forme à partir d'un tableur Excel, vers ArcMap. Le principe étant que la couche des ravinements doit avoir les mêmes systèmes de coordonnées que la carte géoréférencée. De même la couche des surcreusements a été superposée sur celles des sols évoquée plus haut et des classes de pentes obtenues par découpage du Model Numérique de terrain (MNT) de la zone, datant de 2011 ; ceci pour les besoins de l'interprétation de la typologie des formes, par rapport à ces variables.

\section{Résultats et discussion}

\section{Processus et facteurs de formation des surcreusements}

Les ornières sont les traces laissées par l'enfoncement des roues de véhicules sur la piste humide. Pour Egisbeceom (2009), ces sillons longitudinaux présents en permanence dans les bandes de roulement, constituent souvent le point de départ de la dégradation des pistes en terre. En effet, au fur et à mesure que les véhicules roulent sur les mêmes traces, ces sillons s'enfoncent davantage et deviennent des rigoles voire des ravines que les ruissellements approfondissent progressivement (photos 1 et 2). 


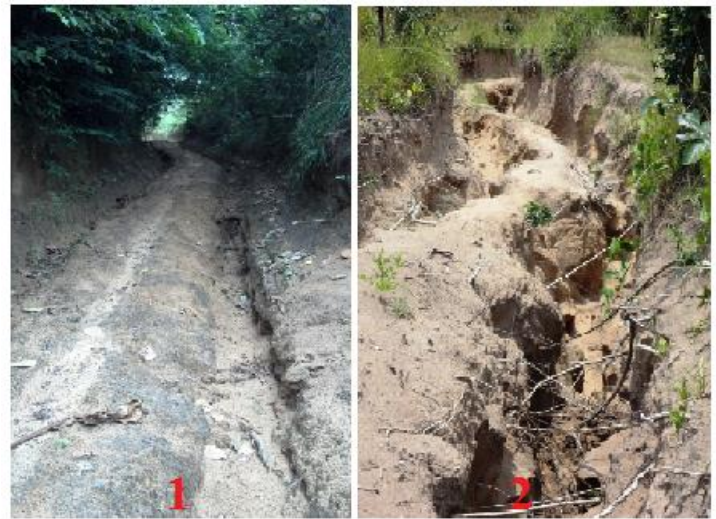

Photo 1 : Piste incisée par l'orniérage vers Mayala ; Photo 2 : Ornières transformées en ravines par les ruissellements sur la piste de Kibossi

(C) Idriss M’bouka Milandou, 2014

Le manque de systèmes de drainage et de domestication des eaux de pluies (canalisations, impluviums, etc.) favorisent l'augmentation du volume des ruissellements qui creusent lesdites ornières.

A partir de 200 millimètres de profondeur sur une section de route de $20 \mathrm{~m}$ de longueur, la piste devient impraticable pour les véhicules de taille moyenne, mêmes les tous terrains (NSW-EPA, 2012), car la bande de terre située entre les ornières constitue un obstacle pour le châssis du véhicule. Ces formes sont à la base de la perte énorme de matériau. Selon Foltz \& Burroughs (1990;1991), lorsque les pistes non revêtues sont affectées par les ornières, elles produisent des quantités de sédiments qui peuvent augmenter de deux à quatre fois par rapport aux routes fraîchement classées (latéritique). A cet effet, lorsqu'une ornière se forme, le ruissellement est empêché de s'écouler à travers la route et est confiné à l'ornière, et le flux concentré provoque une érosion supplémentaire. L'écoulement concentré dans l'ornière continue jusqu'à ce que l'écoulement dépasse l'ornière ou un écoulement croisé soit rencontré. Dans les casgraves, l'ornière peut canaliser l'eau sur de longues distances et contourner les ponceaux de secours ou les drains (Foltz, 1996). Ainsi, l'approfondissement des ornières oblige les automobilistes à effectuer des travaux d'aménagement de la route. La méthode utilisée ici consiste à raser la partie boursouflée encadrée par les deux ornières, pour aplanir la route et permettre le passage des véhicules. Malheureusement, cette méthode contribue à l'approfondissement de la route donc à la mise en place d'autres surcreusements. En effet, quelque soit le tassement de la piste, le processus de formation des ornières reprend une fois la surface de roulement aplanie. En quelques saisons de pluies, le surcreusement se met en place. En raison de l'inclinaison relativement forte de certaines sections de pistes qui atteignent parfois $15 \%$, les ruissellements 
exploitent rapidement les ornières qui se transforment d'abord en rigoles linéaires et ensuite en ravine. Les photos 1 et 2 illustrent bien le processus. Le tronçon delapiste de la photo 2 est abandonné faute de travaux de traitement des ornières. L'aplanissement fréquent de la pistepar les automobilistes contribue à ce processus de surcreusement. Dans la zone d'étude certains surcreusements atteignent $5 \mathrm{~m}$ de profondeur sur près de 500 $\mathrm{m}$ de long (photos $4 ; 5$ et 6 ). Une fois qu'ils sont mis en place, ces formes évoluent verticalement et longitudinalement à cause des travaux d'aplanissement de la piste à chaque dégradation des ornières, et qu'à travers le ravinement par les ruissellements qui «surcreusent» les ornières. Dans ces conditions, deux automobiles ne peuvent rouler dans les deux sens. L'une doit attendre l'autre au bout du surcreusement, en fonction de sa longueur (Photos 7 et 8). Les automobilistes ont eu l'habitude de klaxonner avant de s'engager dans ces tranchées. Ces tranchées formées évoluent aussi latéralement par les mouvements de masse qui se produisent le long des parois généralement abruptes pour les surcreusements rectangulaires (photos $3 ; 5$ et 6 ) et en pente moins abrupte pour les formes trapézoïdales (photo 4). En effet, les parois sont gorgées d'eau pendant les pluies et sous le poids de celle-ci, se produisent souvent des détachements des pans entiers de la paroi qui se déposent sur les pistes. Certains blocs de terre plus volumineux, en atterrissant, forment des bourbiers qui paralysent la circulation en obstruant la piste. Dans ces conditions la section de piste concernée devient impraticable. L'étroitesse des surcreusements n'offrant pas assez de marge de manœuvre (photo 8), les bancs de sables qui s'y accumulent sont la principale cause d'abandon des surcreusements, notamment pendant la saison des pluies. Les automobilistes créent des déviations pour éviter d'y être bloqués. A la fin de la période pluvieuse et au début de la saison sèche, la diminution de l'humidité dans les sols, réduit les effondrements et les parois se stabilisent. Les taux d'argile élevés (plus de 30\%) permettent aux sols de conserver une humidité suffisante qui leur confère une certaine cohésion. 

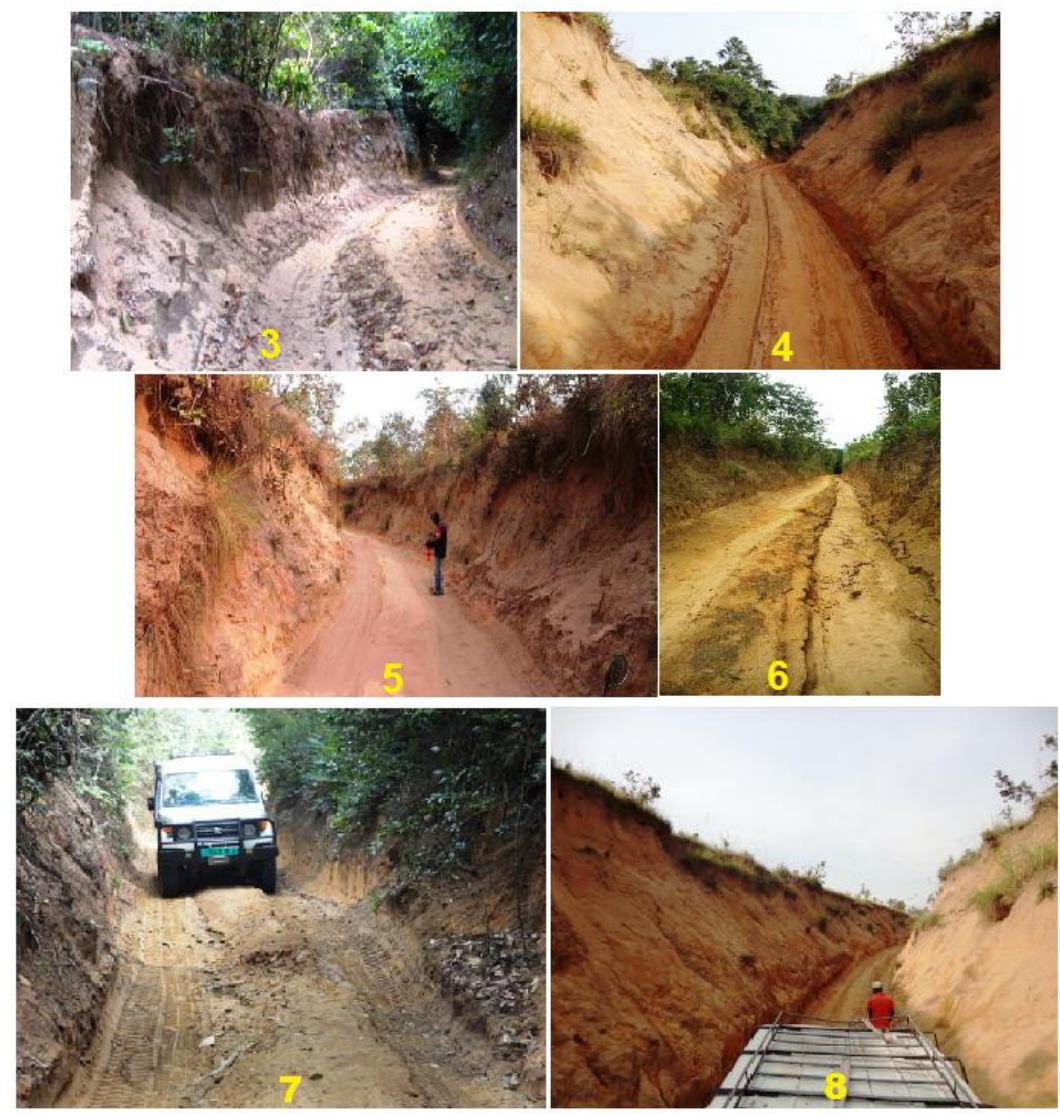

Photos 3 et 6 : Surcreusements rectangulaires sur la piste Koubola-Kibossi ;

Photo 4 : Surcreusement en trapèze sur l'axe Koubola-Kibossi ;Photo 5 :

Surcreusement rectangulaire vers Kibossi ; Photo 7 : surcreusements en formation sur la piste de Mayala ; situation d'un véhicule $4 \mathrm{X} 4$ contraint de suivre les ornières.Photo 8 véhicule coincé dans un surcreusement, sans marge de manœuvre sur la piste de Kibossi (8).

(C) Idriss M'boukaMilandou,2015

$\mathrm{Au}$ milieu de la saison sèche, les argiles asséchées durcissent et servent de liant à l'ensemble de la formation.

Toutefois, le surcreusement est rendu davantage dangereux par le fait que les eaux de ruissellement qu'il canalise, une fois sorties de la tranchée se déversent sur les versants qu'elles décapent ou ravinent avant d'atteindre les cours d'eau qui drainent les fonds de vallées. En effet, ces masses d'eaux qui sortent desdits surcreusements sont responsables d'un ravinement intense des terrains situés à leur débouché. Le rôle des routes dans le déclenchement des érosions a aussi été relevé par Ozer (2014) qui indique que la plupart des ravinements sont provoqués par les infrastructures routières et les pistes piétonnes qui tendent à canaliser les eaux pluviales. Par ailleurs, ces 
ruissellements, produits par les surcreusements, finissent par se déposer les matériaux dans les rivières et les ruisseaux de la zone d'étude qui subissent de ce fait, une sédimentation responsable de l'exhaussement ou rétrécissement de leur lit, ainsi que d'une pollution des eaux affectant la vie aquatique.

A cet effet, Poesen et al.(2003) ; Avni(2005 ); De Vente and Poesen, (2005); Vanmaercke et al.(2011a, 2012b) cités par Vanmaercke et al. (2016), pensent que les ravinements, quels qu'ils soient, sont souvent une source majeure de dépôts sédimentaires dans les bassins versants dans lesquels ils impactent négativement le fonctionnement hydrologique. Ces processus et ces facteurs influent sur les volumes des surcreusements et les pertes en terres occasionnées.

\section{Volumes des vides et des pertes en terre engendrés par les surcreusements}

Sur la piste Koubola-Kibossi, les surcreusements rectangulaires sont plus nombreux, soit 5 sur 8 inventoriés. Mais bien que moins répandues, les formes en trapèze représentent un volume deux fois plus important que les formes précédentes (SP/REC), soit au total $8571,9 \mathrm{~m}^{3}$ contre $4608,29 \mathrm{~m}^{3}$ pour les surcreusements en rectangle. Il importe de noter que la forme trapézoïdale a sa partie supérieure plus large que pour celle de l'autre type. Cette différence s'explique sans doute par un léger déséquilibre entre les taux des sables et des argiles. En effet, une plus grande domination des sables dans les horizons supérieurs peut conférer au matériel une certaine pulvérulence responsable des mouvements de masse de type reptation qu'on observe dans les parties supérieures de certains surcreusements. Une étude plus fine de ce paramètre permettrait de mieux cerner les causes de cette différence d'évolution. Quoi qu'il en soit, le volume total des surcreusements sur cette piste est estimé à $13180,19 \mathrm{~m}^{3}$, avec une perte totale 23724,342 Tonnes. Les surcreusements les plus volumineux sont ceux du PK8 avec près de $5609,85 \mathrm{~m}^{3}$ et 9800 Tonnes de pertes, ainsi que celui du PK2 en trapèze, avec près de $2350 \mathrm{~m}^{3}$ et 4113,65 tonnes de matériaux perdus (Tableau 2).

Tableau 2: Volumes et pertes en terre sur la piste Koubola-Kibossi

\begin{tabular}{|c|c|c|c|c|c|c|c|c|}
\hline \multirow[t]{2}{*}{$\begin{array}{c}\text { Surcreuseme } \\
\mathrm{nt}\end{array}$} & \multirow{2}{*}{$\begin{array}{l}\mathrm{PK} \\
(\mathrm{km} \\
)\end{array}$} & \multirow[t]{2}{*}{$\begin{array}{l}\text { Coordonnées } \\
\text { géographiques }\end{array}$} & \multirow{2}{*}{$\begin{array}{c}\mathrm{L} \\
(\mathrm{m} \\
)\end{array}$} & \multicolumn{2}{|c|}{$\begin{array}{l}\text { Largeur } \\
(\mathrm{m})\end{array}$} & \multirow[t]{2}{*}{$\begin{array}{l}\mathrm{P} \\
(\mathrm{m})\end{array}$} & \multirow[t]{2}{*}{$\mathrm{V}\left(\mathrm{m}^{3}\right)$} & \multirow{2}{*}{$\begin{array}{c}\text { Perte en } \\
\text { terre } \\
\text { (Tonne) }\end{array}$} \\
\hline & & & & $l_{\mathrm{a}}$ & $\mathrm{l}_{\mathrm{b}}$ & & & \\
\hline SP / REC & 1 & $\begin{array}{c}4^{\circ} 20^{\prime} 02^{\prime} \mathrm{S} / 15^{\circ} 02^{\prime} 54 \\
{ }^{\prime} \mathrm{E}\end{array}$ & $\begin{array}{c}20 \\
0\end{array}$ & \multicolumn{2}{|c|}{3,03} & 1,4 & 848,4 & 1484,7 \\
\hline SP / TPZ & 1 & $\begin{array}{c}4^{\circ} 19^{\prime} 48^{\prime \prime} \mathrm{S} / 15^{\circ} 02^{\prime} 45 \\
\text { '’E }\end{array}$ & $\begin{array}{c}18 \\
0\end{array}$ & $\begin{array}{c}2,4 \\
2\end{array}$ & $\begin{array}{c}2,1 \\
7\end{array}$ & $\begin{array}{c}1,4 \\
8\end{array}$ & 611,39 & $\begin{array}{c}1 \\
069,9325 \\
\end{array}$ \\
\hline SP / REC & 2 & $\begin{array}{c}4^{\circ} 19^{\prime} 36^{\prime} \mathrm{S} / 15^{\circ} 02^{\prime} 31 \\
{ }^{\prime} \mathrm{E}\end{array}$ & $\begin{array}{c}15 \\
0\end{array}$ & \multicolumn{2}{|c|}{3,73} & $\begin{array}{c}0,7 \\
3\end{array}$ & 408,44 & 714,77 \\
\hline
\end{tabular}




\begin{tabular}{|c|c|c|c|c|c|c|c|c|}
\hline SP / TPZ & 2 & $\begin{array}{c}4^{\circ} 19^{\prime} 06^{\prime} \mathrm{S} / 15^{\circ} 02^{\prime} 23 \\
{ }^{\prime} \mathrm{E}\end{array}$ & $\begin{array}{c}32 \\
0\end{array}$ & 3,6 & $\begin{array}{c}2,7 \\
6\end{array}$ & $\begin{array}{c}2,3 \\
1\end{array}$ & $\begin{array}{l}2350,6 \\
6\end{array}$ & 4113,655 \\
\hline SP / REC & 3 & $\begin{array}{c}4^{\circ} 18^{\prime} 34^{\prime \prime} \mathrm{S} / 15^{\circ} 02^{\prime} 25 \\
{ }^{\prime} \mathrm{E}\end{array}$ & $\begin{array}{c}33 \\
0 \\
\end{array}$ & \multicolumn{2}{|c|}{4,26} & $\begin{array}{c}1,3 \\
9\end{array}$ & $\begin{array}{c}1954,0 \\
6 \\
\end{array}$ & 3419,605 \\
\hline SP / REC & 4 & $\begin{array}{c}4^{\circ} 17^{\prime} 59^{\prime \prime} \mathrm{S} / 15^{\circ} 02^{\prime} 11 \\
{ }^{\prime} \mathrm{E}\end{array}$ & 70 & \multicolumn{2}{|c|}{4,18} & $\begin{array}{c}1,3 \\
8\end{array}$ & 403,79 & 706,6325 \\
\hline SP / TPZ & 8 & $\begin{array}{c}4^{\circ} 16^{\prime} 43^{\prime \prime} \mathrm{S} / 15^{\circ} 00^{\prime} 27 \\
{ }^{\prime \prime} \mathrm{E}\end{array}$ & $\begin{array}{c}50 \\
0 \\
\end{array}$ & 6,3 & $\begin{array}{c}2,6 \\
4\end{array}$ & $\begin{array}{c}2,5 \\
1 \\
\end{array}$ & $\begin{array}{c}5609,8 \\
5 \\
\end{array}$ & $\begin{array}{c}9 \\
817,2375 \\
\end{array}$ \\
\hline SP / REC & 10 & $\begin{array}{c}4^{\circ} 13^{\prime} 42^{\prime \prime} \mathrm{S} / 15^{\circ} 00^{\prime} 42 \\
, \mathrm{E}\end{array}$ & $\begin{array}{c}20 \\
0\end{array}$ & \multicolumn{2}{|c|}{2,76} & $\begin{array}{c}1,8 \\
0\end{array}$ & 993,6 & 1738,8 \\
\hline \multicolumn{7}{|c|}{ TOTAL } & $\begin{array}{c}13 \\
180,19\end{array}$ & $\begin{array}{c}23724,34 \\
2 \\
\end{array}$ \\
\hline
\end{tabular}

Notes : SP/TPZ : surcreusement de piste en trapèze; SP/REC : surcreusement de piste rectangulaire

PK : poste kilométrique ; $\mathrm{L}$ : longueur $; \mathrm{l}_{\mathrm{a}}$ : largeur de la paroi $1 ; \mathrm{l}_{\mathrm{b}}$ : largeur de la paroi 2 opposée ; P : profondeur ; V : volume totale

Sur le second tronçon (PKB), ce sont plutôt les formes en trapèze qui affectent plus la piste. Leur volume total est estimé à $8484,64 \mathrm{~m}^{3}$. Les surcreusements les plus importants en terme de volume du vide créé et de pertes en terre sont ceux du PK1, 10 et 8, qui ont respectivement $2000 \mathrm{~m}^{3}$ environs avec 3300 tonnes de matériaux perdus ; $1900 \mathrm{~m}^{3}$ environs de volume avec 3200 tonnes. La totalité des pertes en terre est évaluée à 14 423,871 Tonnes (Tableau 3).

Tableau 3 : Volumes et pertes en terre sur la piste Koubola-Bissinza

\begin{tabular}{|c|c|c|c|c|c|c|c|c|}
\hline \multirow[t]{2}{*}{$\begin{array}{l}\text { Surcreusem } \\
\text { ent }\end{array}$} & \multirow{2}{*}{$\begin{array}{c}\mathrm{PK} \\
(\mathrm{km} \\
)\end{array}$} & \multirow[t]{2}{*}{$\begin{array}{l}\text { Coordonnées } \\
\text { géographiques }\end{array}$} & \multirow[t]{2}{*}{$\begin{array}{c}\mathrm{L}(\mathrm{m} \\
)\end{array}$} & \multicolumn{2}{|c|}{$\begin{array}{l}\text { Largeur } \\
(\mathrm{m})\end{array}$} & \multirow[t]{2}{*}{$\mathrm{P}(\mathrm{m}$} & \multirow[t]{2}{*}{$\begin{array}{c}\mathrm{V} \\
\left(\mathrm{m}^{3}\right)\end{array}$} & \multirow{2}{*}{$\begin{array}{l}\text { Perte de } \\
\text { sédiment } \\
\text { s (Tonne) }\end{array}$} \\
\hline & & & & $l_{a}$ & $l_{b}$ & & & \\
\hline SP / TPZ & 1 & $\begin{array}{c}4^{\circ} 20^{\prime} 41^{\prime \prime} \mathrm{S} / 15^{\circ} 02^{\prime} 4 \\
8{ }^{\prime} \mathrm{E}\end{array}$ & 150 & $\begin{array}{c}5,8 \\
3\end{array}$ & $\begin{array}{c}2,3 \\
6\end{array}$ & $\begin{array}{c}3,2 \\
8\end{array}$ & $\begin{array}{c}2014,7 \\
4\end{array}$ & $\begin{array}{c}3324,32 \\
1\end{array}$ \\
\hline SP / TPZ & 1 & $\begin{array}{c}4^{\circ} 20^{\prime} 53^{\prime \prime} \mathrm{S} / 15^{\circ} 02^{\prime} 5 \\
5^{\prime \prime} \mathrm{E}\end{array}$ & 230 & $\begin{array}{c}4,3 \\
5\end{array}$ & $\begin{array}{c}2,5 \\
4\end{array}$ & $\begin{array}{c}1,9 \\
9\end{array}$ & $\begin{array}{c}1576,7 \\
8\end{array}$ & $\begin{array}{c}2601,68 \\
7\end{array}$ \\
\hline SP / TPZ & 2 & $\begin{array}{c}4^{\circ} 21^{\prime} 21^{\prime \prime} \mathrm{S} / 15^{\circ} 03^{\prime} 1 \\
\text { 7'’E}\end{array}$ & 90 & $\begin{array}{c}5,3 \\
5\end{array}$ & $\begin{array}{c}2,5 \\
5\end{array}$ & $\begin{array}{c}2,2 \\
9\end{array}$ & 814,09 & $\begin{array}{c}1343,24 \\
85\end{array}$ \\
\hline SP / TPZ & 3 & $\begin{array}{c}4^{\circ} 21^{\prime} 27^{\prime \prime} \mathrm{S} / 15^{\circ} 03^{\prime} 5 \\
4 \text { '’E }\end{array}$ & 190 & $\begin{array}{c}3,1 \\
8\end{array}$ & $\begin{array}{c}2,4 \\
4 \\
\end{array}$ & $\begin{array}{c}0,9 \\
4 \\
\end{array}$ & 501,87 & 828,0855 \\
\hline SP / TPZ & 8 & $\begin{array}{c}4^{\circ} 22^{\prime} 34^{\prime \prime} \mathrm{S} / 15^{\circ} 06^{\prime} 1 \\
1, ' \mathrm{E}\end{array}$ & 270 & $\begin{array}{c}3,9 \\
7\end{array}$ & $\begin{array}{c}3,1 \\
3 \\
\end{array}$ & $\begin{array}{c}1,6 \\
6 \\
\end{array}$ & $\begin{array}{c}1591,1 \\
1 \\
\end{array}$ & $\begin{array}{c}2625,33 \\
15 \\
\end{array}$ \\
\hline SP / TPZ & 10 & $\begin{array}{c}4^{\circ} 23^{\prime} 15^{\prime} \mathrm{S} / 15^{\circ} 07^{\prime} 0 \\
8^{\prime \prime} \mathrm{E}\end{array}$ & 180 & $\begin{array}{c}5,6 \\
7\end{array}$ & $\begin{array}{c}2,9 \\
5\end{array}$ & $\begin{array}{c}2,5 \\
6\end{array}$ & $\begin{array}{c}1986,0 \\
4\end{array}$ & $\begin{array}{c}3276,96 \\
6\end{array}$ \\
\hline & & TOTAL & & & & & $\begin{array}{c}8484,6 \\
3\end{array}$ & $\begin{array}{c}14423,8 \\
71\end{array}$ \\
\hline
\end{tabular}

$\mathrm{Au}$ regard de ces résultats, les pertes en terre occasionnées par ce phénomène sont énormes. Certes à l'échelle de chaque surcreusement, ces pertes sont modestes en comparaison avec les 24065,3 tonnes de terre érodées par un ravinement kilométrique trouvés par Hughes et al. (2001) en 
Australie, cependant sur les deux pistes, l'ensemble des pertes occasionnées par tous les surcreusements étudiés est estimé à 38 148,213 tonnes. Plusieurs auteurs, notamment Poesen et al.(2003) ; Valentin et al., (2005 ) et Makanzu Imwangana (2014) cités par Vanmaercke et al.(2016), affirment que le développement des ravinements occasionne une perte énorme des terres et affecte beaucoup les infrastructures, comme les routes de Koubola-Kibossi et Koubola-Bissinza. Ce phénomène s'explique par la sensibilité du milieu visà-vis de l'érosion.

\section{Sensibilité des surfaces des pistes surcreusées}

Le tableau 4 montre les caractéristiques physiques de la surface de roulement des pistes Koubola-Kibossi (PKK) et Koubola-Bissinza(PKB)

Tableau 4 : caractéristiques physiques des deux chaussées

\begin{tabular}{|c|c|c|c|c|}
\hline Piste & LMP $(\mathrm{m})$ & $\begin{array}{c}d a \\
\left(\mathrm{~g} / \mathrm{cm}^{3}\right)\end{array}$ & $\begin{array}{c}\text { Porosité } \\
(\%)\end{array}$ & $\begin{array}{c}\text { ESR } \\
(\mathrm{cm})\end{array}$ \\
\hline PKK & 3 & 1,8 & 20,33 & 0 \\
\hline PKB & 2,5 & 1,7 & 33,5 & 0 \\
\hline
\end{tabular}

Notes : MR : matériel remanié ; LMP : largeur moyenne des pistes ; $d a$ : densité apparente ; ESR : épaisseur $A=\pi r^{2}$ de roulement.

Les résultats montrent que sur la piste Koubola-Kibossi, la $\boldsymbol{d a}$ est relativement forte avec $1,8 \mathrm{~g} / \mathrm{cm}^{3}$; la porosité est relativement faible de $20,33 \%$. Ces données sont conformes aux normes selon lesquelles, plus la densité apparente est forte, plus la porosité est faible, et vice-versa. Ils indiquent que la surface de cette piste est tassée. Dans ces conditions, l'eau de pluies ruisselle beaucoup plus qu'elle s'infiltre. Par contre, la piste Kibossi-Bissinza est moins tassée par rapport à la première : la $\boldsymbol{d a}$ est légèrement plus faible $\left(1,7 \mathrm{~g} / \mathrm{cm}^{3}\right)$ et la porosité plus élevée (34\%).Cela signifie que l'infiltration est un peu plus importante au détriment des ruissellements. A ces données liées à la densité apparente et à la porosité du MR des deux pistes, s'ajoute la largeur des deux pistes estimées à 2,5 et $3 \mathrm{~m}$. En effet, plus la largeur de la route qui correspond à celle du surcreusement est petite, plus les ruissellements sont concentrés et causent plus de dégâts érosifs.

L'étroitesse de ces pistes est donc un facteur de vulnérabilité dans la mesure où les ruissellements s'étalent sur toute la surface de la piste. En l'absence des canalisations où ils auraient pu se concentrer, ces écoulements d'eau ravinent la piste de roulement.

\section{Texture des sols autour des pistes surcreusées}

La texture des formations géologiques est également un facteur de fragilité de ces axes routiers. Les résultats de ces variables sont consignés dans le tableau 5. 
Tableau 5 : texture et matière organique des formations géologiques autour des deux pistes

\begin{tabular}{|c|c|c|c|c|c|c|c|}
\hline \multirow{3}{*}{ Piste } & \multirow{3}{*}{$\begin{array}{l}\text { Profondeur } \\
\text { échantillon }\end{array}$} & \multicolumn{5}{|c|}{ Texture moyenne (\%) } & \multirow{3}{*}{ MO } \\
\hline & & \multicolumn{2}{|c|}{ Sables } & \multicolumn{2}{|c|}{ Limons } & \multirow[t]{2}{*}{ Argile } & \\
\hline & & SF & SG & LF & LG & & \\
\hline \multirow{3}{*}{ PKK } & $15 \mathrm{~cm}$ & 25,0 & 28,5 & 5,1 & 2,9 & 35,0 & 1,1 \\
\hline & $50 \mathrm{~cm}$ & 24,7 & 25,9 & 8,2 & 7,5 & 30,9 & 2,0 \\
\hline & $100 \mathrm{~cm}$ & 28,4 & 38,1 & 4,2 & 2,3 & 24,0 & 2,1 \\
\hline \multirow{3}{*}{ PKB } & $15 \mathrm{~cm}$ & 20,2 & 34,2 & 1,4 & 1,0 & 38,1 & 5,0 \\
\hline & $50 \mathrm{~cm}$ & 16,2 & 33,6 & 3,7 & 5,3 & 39,2 & 1,9 \\
\hline & $100 \mathrm{~cm}$ & 17,2 & 37,1 & 5,2 & 4,1 & 32,2 & 4,0 \\
\hline
\end{tabular}

Notes : SF : sable fin ; SG : sable grossier ; LF : limon fin ; LG : limon grossier ; $\mathrm{MO}$ : matière organique

Ces résultats montrent que dans l'ensemble la texture des formations géologiques situées le long des pistes est sablo-argileuse avec des taux d'argile moyens de 33\%. Ces taux d'argile qui dépassent les $30 \%$ dans tous les niveaux de profondeurs ou presque et l'importance des sables fins confèrent à ces formations une certaine cohésion qui réduit leur affouillabilité vis-à-vis des ruissellements. La cohésion du matériel est renforcée par une teneur en matière organique relativement notoire surtout le long de la seconde piste (Tableau 4). Il importe de noter que c'est la présence notable des argiles dans les formations superficielles qui est le principal paramètre qui favorise la formation des surcreusements. Elle confère au matériau la cohésion nécessaire à la verticalité des parois qui constitue la condition sine qanun d'évolution vers le surcreusement. La localisation de ces formes est en rapport avec ces caractéristiques géologiques comme il est montré plus haut. Mais cette texture est en rapport avec les taux d'érodibilité trouvés sur le terrain. En effet, les résultats des mesures granulométriques sont conformes aux valeurs d'indices d'érodibilité (K) trouvés par M'bouka Milandou et al. (2016). Ils sont estimés à 0,10 t.ha.h/ha.MJ.mm pour le secteur de la piste Koubola-Bissinza et à 0,20 pour celui de l'axe Koubola-Bissinza. Situés à l'intervalle de 0,10 à 0,25 t.ha.h/ha.MJ.mm;ils confèrent aux sols des deux secteurs une résistance faible à l'érosion selon Bollinne et Rosseau (1978).A l'inverse, cette abondance des liants diminue les infiltrations au profit des ruissellements. L'importance des volumes et leur concentration liée à l'étroitesse des surfaces de roulement donnent aux ruissellements une grande capacité de ravinement. Ces ruissellements sont dus à l'agressivité des pluies sur le plateau des Cataractes.

\section{L'agressivité des pluies}

Ce phénomène est aussi entretenu par une agressivité des pluies très marquée. Le tableau 6 et la figure 2 montrent les résultats obtenus après 
calcul de l'indice d'érosivité $(\mathrm{R})$ des pluies à partir des moyennes annuelles de précipitation à Brazzaville et les environs entre 2005 et 2014.

Tableau 6: érosivité interannuelle des pluies entre 2005 et 2014 à la station de Maya-Maya

\begin{tabular}{|c|c|c|c|c|c|c|c|c|c|c|c|}
\hline Année & 2005 & 2006 & 2007 & 2008 & 2009 & 2010 & 2011 & 2012 & 2013 & 2014 & Moyenne \\
\hline $\begin{array}{c}\mathrm{P} \\
(\mathrm{mm})\end{array}$ & 1326 & $\begin{array}{c}1 \\
364,8\end{array}$ & 1701,6 & $\begin{array}{c}1 \\
642,8\end{array}$ & $\begin{array}{c}1 \\
539,2\end{array}$ & 1329,7 & $\begin{array}{c}1 \\
675,7\end{array}$ & 1198,4 & 1596,6 & 1293,7 & 1466,85 \\
\hline $\mathrm{R}$ & 6189,13 & $\begin{array}{c}6 \\
570,41\end{array}$ & 10399,3 & $\begin{array}{c}9 \\
663,8\end{array}$ & $\begin{array}{c}8 \\
436,82\end{array}$ & 6224,95 & $\begin{array}{c}10 \\
071,84\end{array}$ & 5022,4 & 9105,72 & 5881,15 & 7756,55 \\
\hline
\end{tabular}

Notes : R : paramètre d'érosivité et $\mathrm{P}:$ précipitation annuelle $(\mathrm{mm})$

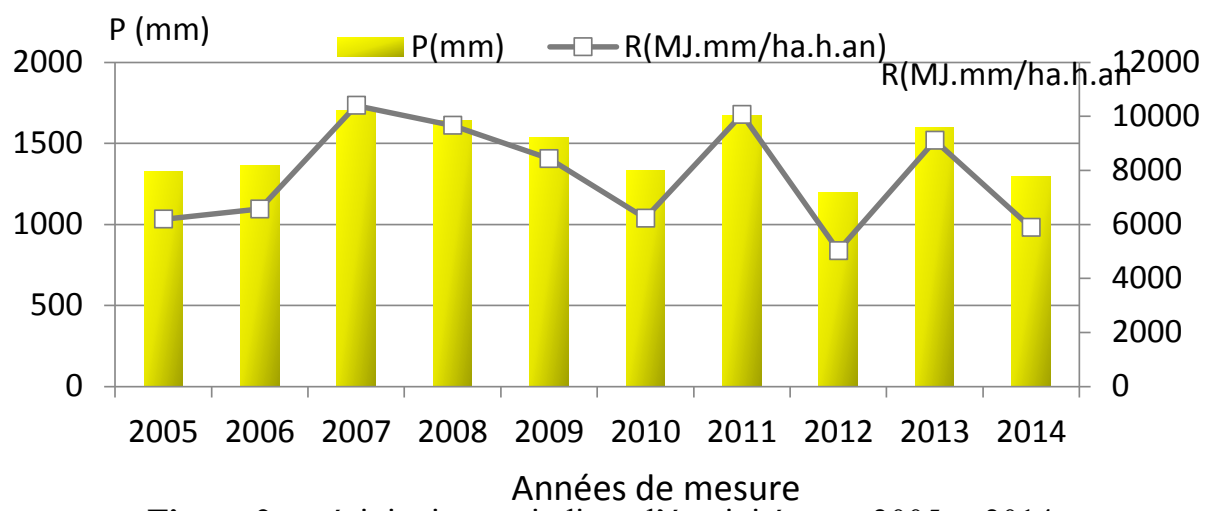

Figure 2 : précipitations et indices d'érosivité entre 2005 et 2014

Ce diagramme combinant les précipitations annuelles et l'indice $\mathrm{R}$ de 10 ans, montre qu'en premier lieu, qu'avec une hauteur moyenne annuelle de $1400 \mathrm{~mm}$, les pluies sont abondantes. Cela se confirme avec les moyennes mensuelles qui dépassent souvent les $100 \mathrm{~mm}$ et varient entre $180 \mathrm{~mm}$ et $260 \mathrm{~mm}$ pendant les mois les plus humides (novembre, décembre, mars et avril) (M'bouka Milandou et al., 2016). Cette variation interannuelle des moyennes pluviométriques influe sur l'érosivité des pluies. Ce qui fait que plus l'année est pluvieuse plus l'érosivité augmente. Les années 2007 ; 2011 et 2013 ont une érosivité plus forte avec des indices $>8000 \mathrm{MJ} . \mathrm{mm} / \mathrm{ha} . \mathrm{h} . \mathrm{an}$, pour près de $1600 \mathrm{~mm}$ des hauteurs de pluies dans l'ensemble. Secundo, la moyenne de l'indice R, en 10 ans, est estimée à 7 756,55 MJ.mm/ha.h.an. Il dépasse le seuil de 5000 MJ.mm/ha.h.an que Douay et Lardieg (2010) définissent comme une érosivité très forte (Tableau 6). L'indice $\mathrm{R}$ de 8 années (2005-2011 et 2013) sur les 10 évaluées, dépasse les 5000 MJ.mm/ha.h.an et est largement supérieur à celui trouvé en Côte d'Ivoire, estimé à $1260 \mathrm{MJ} . \mathrm{mm} / \mathrm{ha}$.h.an, pour $2100 \mathrm{~mm}$ de pluie. Cette élévation prouve le caractère agressif du climat de la zone tropicale humide (Roose et Lelong, 1976), responsable de l'érosivité des pluies qui participe largement à la genèse et à l'évolution des surcreusements de pistes rurales ou forestières. 
L'agressivité des pluies influe sur la densité des surcreusements, grâce à la sensibilité des sols et des pentes autour des 2 pistes. Pour ce faire, une analyse cartographique a été effectuée pour illustrer cet aspect.

\section{Analyse cartographique des surcreusements}

La cartographie a permis, d'une part, de montrer la densité des surcreusements sur les deux pistes et d'autre part, de localiser lesdites formes par rapport à la nature des formations géologiques en place et le relief. Du point de vue de leur répartition, la piste Koubola-Kibossi est beaucoup plus affectée que la piste Koubola-Bissinza, soit 8 surcreusements sur le premier tronçon contre 6 sur le second (Figure 3).

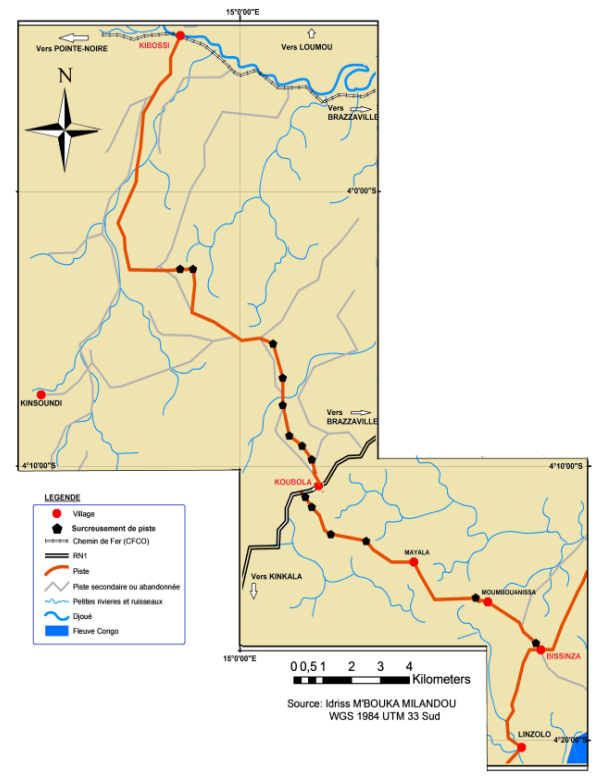

Figure 3 : Localisation des surcreusements étudiés

En termes de densité, c'est-à-dire du nombre de surcreusements au kilomètre, les deux pistes ont des valeurs presqu'identiques, soit 0.53 pour le premier et 0.54 pour le second tronçon. Cependant, on note une nette concentration du phénomène dans la partie sud de la zone d'étude, c'est-àdire dans la moitié sud de la piste nord et presque sur toute la piste sud. Cette concentration est plus importante encore dans la partie centrale. Au nord de la première piste le phénomène est absent alors qu'au sud de la seconde piste la concentration est moins importante. Cette localisation semble bien être liée à la nature des roches en place (Figure 3). La superposition de la carte des pistes et des sols que nous avons réalisées montre bien que ces formes typiques se localisent dans les secteurs où affleurent les grès de l'Inkisi 
(Figure 4).Sur ces roches, les formations superficielles affectées par le phénomène sont des altérites à forte concentration d'argile qui sont les éléments favorisant le maintien de la verticalité des parois des tranchées. C'est le domaine des sols ferralitiques fortement désaturés que Denis(1974) avait décrits sur une surface couvrant les localités ouest de Brazzaville, jusqu'à Kinkala, en passant par Goma tsé-tsé.

Ces formes semblent absentes dans les domaines sablonneux ou à dominance sableuse où la pulvérulence du matériel ne permet pas la formation de parois verticales. Mais la topographie du site semble avoir aussi joué un rôle notoire, car les sections des pistes affectées se localisent soit sur les sommets de collines, les versants et les flancs de vallées. Ici, il a été constaté que presque toutes les sections «surcreusées 》 se situent à l'intervalle des pentes de 15 à $25^{\circ}$ et beaucoup plus de $25^{\circ}$ à plus, ce qui engendre plus de pertes de terre (Fig. 5)

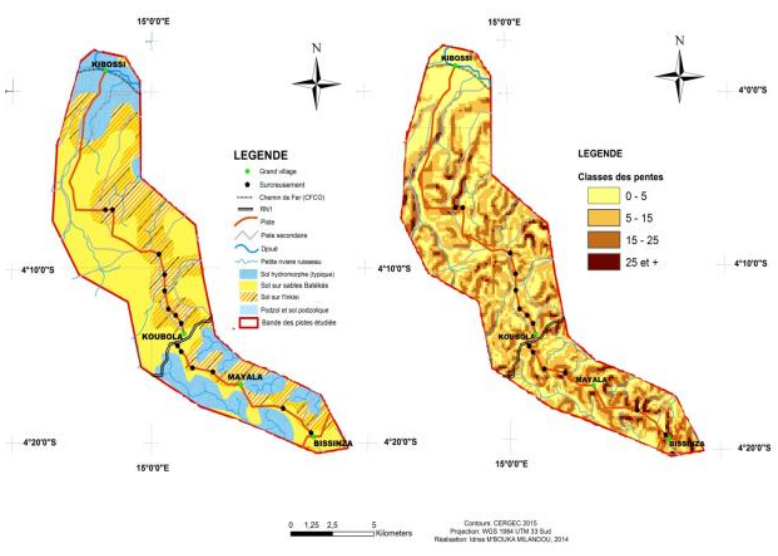

Figure 4 : tracé des pistes et affleurements géologiques.

Figure 5 : le tracé des pistes et le relief.

Cela montre que la topographie est un facteur important de l'érosion; la variation des pentes influence directement la genèse et l'évolution de tout type de ravinements à travers le degré de pente qui contrôle directement les ruissellements pluviométriques (Vanmaercke et al., 2016). Ce phénomène affecte largement la fluidité de transport, par des retards de la circulation automobile, pour l'écoulement des produits agricoles, de pèches, de chasse, de l'élevage et de l'exploitation forestières vers Brazzaville. A cet effet, deux automobiles ne peuvent circuler dans les deux sens, l'un doit attendre l'autre et avant de s'engager dans la tranchée, les automobilistes doivent klaxonner pour éviter de bloquer la circulation. 


\section{Proposition des solutions de traitement des surcreusements}

Les solutions nécessitent une étude de faisabilité approfondie. Toutefois elles sont à la fois préventives et curatives.

\section{Proposition des solutions préventives}

Les surcreusements de pistes peuvent être évités en corrigeant le traitement des ornières qui favorise le phénomène de surcreusement. Au lieu d'aplanir les rides entre les ornières, il faudrait plutôt remblayer les ornières, dès leur formation, avec de la latérite et couvrir ensuite la piste d'une couche traitée. Le but est d'éviter le processus d'aggravation des ornières et du début de surcreusement. Ensuite, la piste doit être protégée, en construisant des systèmes de drainages (canalisation, impluvium, etc.) des eaux de pluies, pour la maitrise des ruissellements et enfin concevoir un plan d'entretien régulier.

\section{Proposition des solutions curatives}

Dans le cas des surcreusements déjà formés, plusieurs solutions sont possibles parmi les quelles deux peuvent être retenues.

- $\quad$ La première est le colmatage, c'est-à-dire, le remblaiement de la tranchée pour réduire sa profondeur. La technique consisterait à remplir d'abord la forme avec des déchets solides de toutes sortes, traités, trié et compactés par des engins appropriés. Cette première couche doit être couverte par la suite par la latérite. Couvrir enfin la piste d'une couche traitée et la protéger par un système de drainages (canalisation, impluvium, etc.) des eaux de pluies. Mais il importe de relever que cette opération est onéreuse et nécessite des moyens financiers et matériels (tracteur, poklins, etc.) dont seul l'Etat peut disposer. Le nombre de surcreusement à traiter rend cette éventualité encore plus difficile à envisager. Mais l'importance socioéconomique des pistes concernées est un facteur qui peut influer sur l'intervention des pouvoirs publics au niveau national ou départemental.

- La deuxième solution est la stabilisation des parois des tranchées. Celle-ci peut être réalisée soit par bétonnage soit par la combinaison de deux techniques antiérosives : la méthode du béton projeté et la méthode d'armement du sol par des poutrelles. Ces méthodes ont été utilisée sur l'axe Igné-Mindouli et la section réhabilité Dolisie- Pointe-Noire dans le Mayombe (Photos 7A et B). 

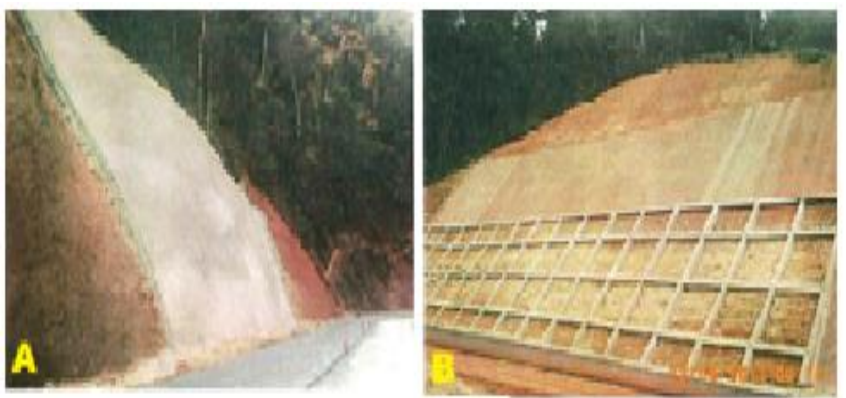

Photos 7 : A- talus stabilisé par la technique de béton ; B-Talusstabilisé par la méthode du béton projeté et la méthoded'armement dusol par des poutrellessur la nationale $\mathrm{N}^{\circ} 1$

Pointe-Noire / Brazzaville (C) Bassoukissa (2012)

La stabilisation des parois nécessite d'abord l'adoucissement de leur pente trop abrupte. Les parois des formes en rectangle doivent être taillées en trapèze et celles des formes en trapèze doivent être adoucies davantage (Figure 6).

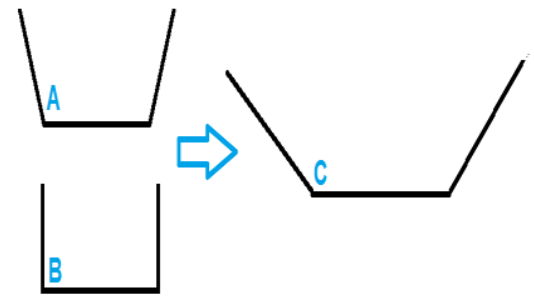

Figures 6: A : profil transversal d'un surcreusement en trapèze avec des parois inclinées ; B : Surcreusement rectangulaire aux parois abruptes; $\mathrm{C}$ : forme d'adoucissement des parois avant la végétalisation.

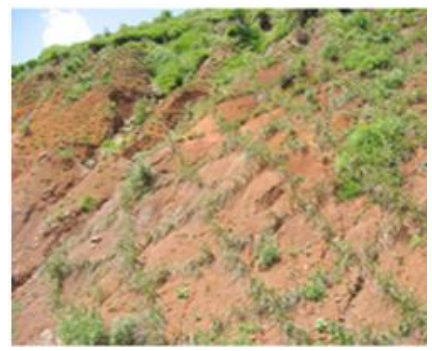

Photo 8: applications of Vetiver grass for landscape stabilization. (C) GTZ-IFSP/ South Gonder, 2005)

Après l'adoucissement des parois, à défaut de les bétonner, on peut appliquer les méthodes biologiques. Les plantes les plus efficaces dans la stabilisation des talus routiers sont les vétivers. Les végétaux doivent être disposés de façon entrecroisés (Photo 8). Cela permettra également de stabiliser la forme et éviter son évolution latérale. Le vétiver a déjà fait ses preuves à Brazzaville (Ndona et Truong, 2007) et sur la bretelle bitumée Igné - Mindouli (RN1), car ils s'adaptent à tout type de sols (sableux, argileux, sablo-argileux, argileux-sableux, etc.) comme ceux des deux secteurs de piste. Leurs racines profondes stabilisent et restaurent les secteurs affectés.

\section{Conclusion}

Le surcreusement des pistes est un phénomène qui semble se répandre dans le département du Pool particulièrement sur le plateau des Cataractes, au sud de Brazzaville. 
Ces formes sont certes l'œuvre des ruissellements qui approfondissent les ornières de la piste, mais c'est la méthode de traitement de ces rigoles qui est le facteur principal de déclenchement du processus. La sensibilité de la surface de roulement, liée à la texture, la structure et la forte densité du sol, la topographie et l'agressivité pluviométrique, sont autant de facteurs qui, associés à l'absence de système de drainage, influent sur les ruissellements responsables la mise en place de ces formes particulières. L'impact négatif de ce type d'érosion sur la circulation des hommes et des biens, d'une part et l'accélération de l'érosion et la pollution des rivières qu'il engendre, d'autre part, constituent une grande préoccupation. Il importe par conséquent que des études soient entreprises sur la recherche des solutions. A ce niveau un regard devrait être porté sur la principale méthode de traitement des ornières, responsable du déclenchement des processus de formation. Pour les surcreusements déjà formés l'accent devrait être mis sur les méthodes de stabilisation des parois et sur les moyens de la maîtrise des écoulements d'eau à l'intérieur du surcreusement. Il importe de noter que si aucune solution n'est trouvée pour la stabilisation des parois et la maîtrise des eaux dans ces tranchées, ces pistes seront de plus en plus surcreusées, avec des impacts négatifs sur le transport des personnes et de leurs biens, ainsi que des problèmes de pollution des cours d'eau.

\section{References:}

1. Bollinne A., Rosseau P., 1978 :« Erodibilité des sols de moyenne et haute Belgique. Utilisation d'une méthode de calcul du facteur $K$ de l'équation universelle de perte en terre ».Bull. Soc. Géogr. de Li\&ge, $14,4:$ p.127-140.

2. Denis B., $1974:$ :Notice explicative $n^{\circ} 52$; carte pédologique au 1/20.000 de Brazzaville-Kinkala, République populaire du Congo », Paris, ORSTOM, 101 p.+cartes ;

3. Descoings B., 1975: «Les grandes régions naturelles du Congo, Candollea 30. P. 91-120;

4. Douay D. et Lardieg E. 2010 : Méthodologie de cartographie de la vulnérabilité intrinsèque des captages d'eau superficielle ». ANTEA, GINGER et GALLIGGE / Agence de l'eau - Adour-Garonne / Ets public de l'état (France).28 p. ;

5. Egisbeceom $2009:$ :Catalogue des dégradations des routes non revêtues ». Ministère de l'Equipement et des Transports. Direction Nationale des Routes (DNR), République du Mali, UE. 30 p. ;

6. Foltz, R. B. 1996: "Traffic and No-Traffic on an Aggregate Surfaced Road: Sediment Production Differences". Proceedings of the Seminar on Environmentally Sound Forest Road and Wood Transport; June 1996; Sinaia, Romania Rome, Italy: FAO. 13 p. 
7. Foltz, R. B. \& Burroughs, E. R., Jr., 1991: "A test of normal tire pressure and reduced tire pressureon forest roads: sedimentation effects". In: Proc. Forestry and Environment...Engineering Solutions, Forest Engineering Group, Amer. Soc. Of Agric. Engrs., New Orleans, Louisiana, USA, p. 103-112.

8. Foltz, R.B. \& Burroughs, E.R., Jr. 1990: "Sediment production from forest roads with wheel ruts". Proceedings from Watershed Planning and Analysis in Action. American Society of Civil Engineers, Durango, Colorado, USA, p. 266-275 ;

9. Hughes, A .O., Prosser, I.P., Stevenson,J.,Scott, A., Lu, H., Gallant, J. and Morgan, C.J., 2001: "Gully erosion mapping for the National Land Water Resources Audit”.Technical Report 26/01, CSRO Land and Water, Camberra, Australia. p.1-18;

10. Makanzu Imwangana F., Dewitte O., Ntombi M., Moeyersons J., 2014: "Topographic and road controlof mega-gullies in Kinshasa (DR Congo)".Geomorphology 217; p.131-139.;

11. M'bouka Milandou I., 2009 : "Etude de l'érosion à Linzolo et ses environs ». Mémoire de Maîtrise. Option géomorphologue ; FLSH, UMNG, 109 p.

12. M’bouka Milandou I., 2013 :« Etude de la dégradation des routes en milieu tropical chaud et humide: cas du Congo Brazzaville ". Mémoire de DEA. Option géomorphologue ; FLASH, UMNG, 99 p.;

13. M'bouka Milandou I, Sitou L. \&Bouzou Moussa I, 2016 : Analyse de l'érodibilité des sols dans la Sous-préfecture de Goma tsé-tsé (République du Congo) ", Revue semestrielle de l'Université de Zinder (Niger) «territoires, société et environnements », $\mathrm{N}^{\circ} 007$, juin 2016 ; p. 43-58;

14. Ndona A. et Truong P.,2007:"Ravine rehabilitation in Brazzaville, Congo".Vetiver Network International Seminary. $15 \mathrm{p}$

15. NSW-EPA, 2012: "Forest infrastructure”.The NSW Environment Protection Authority (EPA).http://www.epa.nsw.gov.au /resources/pnf/ 09761 Chap5PNFFldGdNthnNSW.pdf. 36 p.

16. Ozer P.,2014: "Catastrophes naturelles et aménagement $d u$ territoire; intérêt des images Google Earth dans les pays en développement ». Géo-Eco-Trop., 38,1,n.s. p. 209-220.

17. Roose E.J. et Lelong F., 1976 : Les facteurs de l'érosion hydrique en Afrique tropicale. Etudes sur petites parcelles expérimentales de sol ». Revue de géographie physique et de géologie dynamique (2), Vol. XVIII, Fasc.4, p.363-374;

18. Samba Kimbata M.J., 1978 : Le climat du Bas-Congo, thèse de $3^{\text {ème }}$ cycle », Université de Bourgogne, Dijon, 280 + figures ; 
19. Vanmaercke M, Poesen J., Van Mele B., Demuzere M., Bruynseels A., Golosov V., Bezerra J.F.R., Bolysov S., Dvinskih A., Frankl A., Fuseina Y., Guerra A.J.T., Haregeweyn N., Ionita I., MakanzuImwangana F., Moeyersons J., Moshe I., Samani A.N., Niascu L., Nyssen J., Otsuki Y., Radoane M., Rysin I., Ryzhov Y.V., Yermolaev O., 2016: "How fast do gullyheadcutsretreat ».EarthScience Reviews 154. P. 336-355. 